\title{
EFICACIA LUMINOSA DE LA RADIACIÓN SOLAR GLOBAL PARA SUPERFICIE HORIZONTAL EN MADRID. ESPAÑA
}

\author{
(THE LUMINOUS EFFICACY FOR GLOBAL SOLAR RADIATION FOR HORIZONTAL \\ SURFACES IN MADRID. SPAIN)
}

P. Oteiza*; A. Soler*; G. Yáñez *

\section{RESUMEN}

Cuando no se tienen medidas de iluminación natural, pero se dispone de buenos registros de radiación solar, la eficacia luminosa es un factor muy importante ya que permite obtener una a partir de otra. En este artículo se presentan los resultados de las medidas de la eficacia luminosa de la radiación solar global, efectuadas en Madrid, durante los meses de abril, mayo y junio de 1989, los primeros publicados para una localidad española. Se estudia la dependencia de la eficacia con la nubosidad. Las medidas se comparan con las registradas en otros lugares donde se han realizado estudios sistemáticos. Se estudia la variación de la eficacia con la altura solar para cielo promedio, cielo semicubierto y cielo despejado.

\section{SUMMARY}

When measured daylighting data are not available, but solar radiation is recorded, if values of the luminous efficacy are known, daylighting data can be estimated. In this paper we give the results of measurements of the luminous efficacy of global solar radiation, obtained in Madrid, in April, May and June, 1989, which are the first published for a Spanish location. We study the dependence of the luminous efficacy on cloudiness. Measurements are compared with those undertaken in other location where continuous measurements are available. We study the dependence of luminous efficacy on solar elevation for different degrees of cloudiness.

\section{INTRODUCCIÓN}

La luz del día es una de las variables naturales que más influencia tienen en la percepción de la arquitectura y en el ahorro energético en edificaciones no residenciales. Dada la ausencia de medidas de iluminación natural directas, y actualizadas en España, es de interés obtener una base de datos de iluminancia aunque sea de forma indirecta.

Cuando no se dispone de medidas directas de iluminancia, o éstas son insuficientes, se pueden utilizar métodos indirectos de cuantificación de la luz natu- ral, que permiten deducir la iluminancia cuando se conocen otras variables naturales que están en relación más o menos directa con ella. Aunque ninguno es mejor que la medición directa, pueden darnos una razonable aproximación a la iluminancia real.

Los métodos que predicen la iluminancia a partir de los datos de radiación solar, dado el valor de la eficacia luminosa ${ }^{1}$, son probablemente los más versátiles de aplicar ya que son sensibles a variaciones en el clima y la latitud. Se basan en medidas de la irradiancia solar que se realizan habitualmente en la red meteorológica nacional de muchos países.

\footnotetext{
*Departamento de Física e Instalaciones. * *Departamento de Proyectos Arquitectónicos, Universidad Politécnica de Madrid. Escuela Técnica Superior de Arquitectura. Avda. Juan de Herrera, 4. 28040 Madrid.
} 
Por lo que respecta a España, es uno de los países europeos que posee menos información sobre los valores de iluminación natural, a pesar de las recomendaciones de la Comisión Internacional de la Iluminación (CIE), que ha expresado reiteradamente la conveniencia de realizar este tipo de mediciones. Las únicas medidas en España, de las que tenemos constancia, son las realizadas en Madrid, en $1955^{2}$. Es conocido que los valores de la iluminación natural pueden variar para un lugar con el paso del tiempo, debido a cambios en los diversos parámetros atmosféricos que la afectan. Dicha variación puede ser importante en un ambiente urbano ${ }^{3}$. Por este motivo se realizaron medidas de la iluminancia global sobre superficie horizontal para Madrid, durante el año $1989^{4}$.

No existen valores de la eficacia luminosa para poblaciones españolas. Por ello se realizaron simultáneamente a las medidas de iluminancia global medidas de irradiancia solar global. En este artículo presentamos los valores obtenidos para la eficacia luminosa sobre superficie horizontal, tal y como se define en la sección siguiente.

\section{EFICACIA LUMINOSA}

La magnitud que relaciona la iluminancia y la irradiancia de una fuente energética se denomina eficacia luminosa $\mathrm{K}, \mathrm{y}$ se mide en lúmenes/vatio, $\mathbf{I m} \mathbf{W}^{-1}$.

La eficacia luminosa $\mathrm{K}$, de la radiación solar se puede expresar como la relación entre la iluminancia, $E_{v}$, y la irradiancia $E_{e}$, correspondiente a dicha radiación ${ }^{5}$ :

$$
K=\frac{E_{v}}{E_{e}}
$$

Sin embargo, hay que tener en cuenta que la eficacia luminosa no es una constante, ya que se ha demostrado que varía con la altura solar, con la cobertura de nubes y la cantidad de aerosoles y vapor de agua de la atmósfera, y varía también de acuerdo a si es difusa, directa o global la radiación que esté siendo considerada ${ }^{1}$.

Las medidas de la eficacia luminosa que presentamos en este trabajo para Madrid, se refieren a valores de la iluminancia global y la irradiancia global, para superficies horizontales. Littlefair ${ }^{1,6}$ en amplios estudios referidos a la eficacia luminosa, muestra su variabilidad y las relaciones que la vinculan con otras variables ambientales. En el primero de sus trabajos' ${ }^{1}$, que fue publicado en 1985, hace una recopilación de las mediciones y los cálculos teóricos obtenidos por distintos autores en varias partes del mundo. El segundo, publicado en $1988^{6}$, presenta los resultados de un año de mediciones de la eficacia luminosa en Garston (Gran Bretaña).

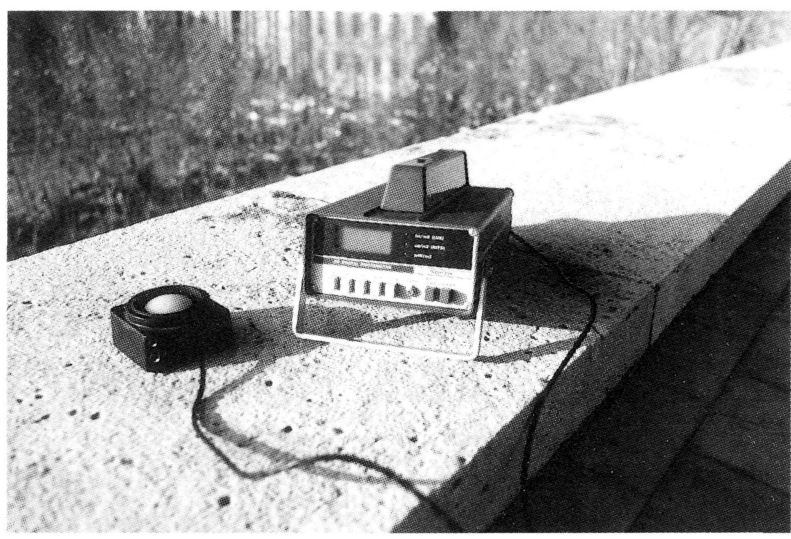

A

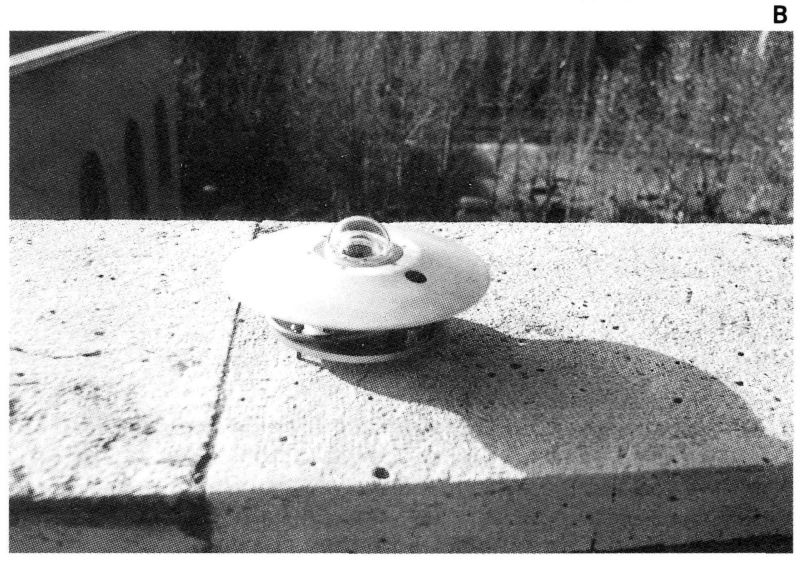

Fig. 1. - Instrumentos de medida utilizados en Madrid: A) Luxómetro para medir iluminancia, con sensores para medidas en el exterior $y$ en el interior. B) Piranómetro para medir irradiancia solar.

\section{MEDIDAS DE EFICACIA LUMINOSA DE LA RADIACIÓN SOLAR GLOBAL PARA SUPERFICIE HORIZONTAL EN MADRID}

Para obtener experimentalmente valores de eficacia luminosa de la radiación solar global en Madrid $\left(40,29^{\circ} \mathrm{N}, 4,42^{\circ} \mathrm{W}, 710 \mathrm{~m}\right.$ de altitud) se tomaron medidas simultáneas de iluminancia global e irradiancia global. Las mediciones se realizaron en la azotea de un edificio de cuatro pisos del Instituto de Óptica "Daza de Valdés", perteneciente al Consejo Superior de Investigaciones Científicas de Madrid; en el mismo lugar donde se efectuaron las medidas de iluminancia, en el año $1955^{2}$. El período de toma de datos fue de tres meses: abril, mayo y junio de 1989. Se tomaron medidas a las 9 y 12 horas, del Tiempo Solar Verdadero.

Las mediciones se realizaron sobre plano horizontal. Junto con las medidas, se registraron también las condiciones del cielo: Despejado, Semicubierto y Cubierto. Caracterizándose como "Despejado" un cielo con ausencia tota| de nubes: "Cubierto" un cielo con nu- 
12:00 Horas

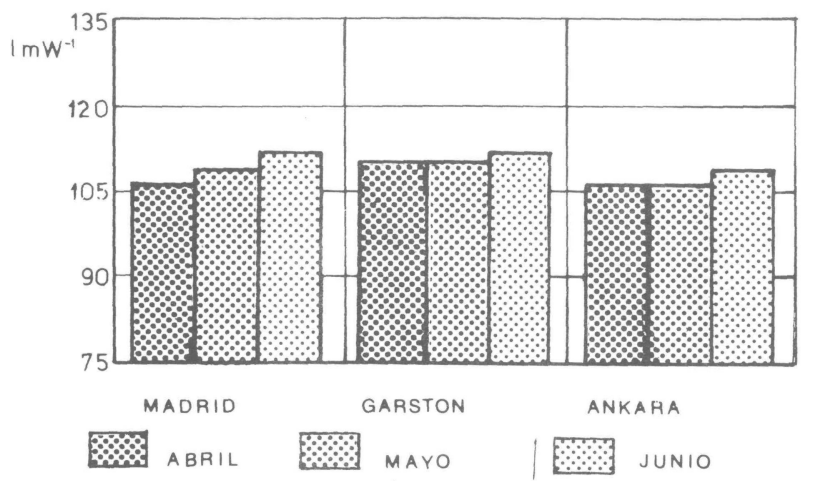

Flg. 2. - Eficacia luminosa de la radiación solar global, con cielo promedio, para Madrid y otras dos ciudades donde se han realizado mediciones similares.

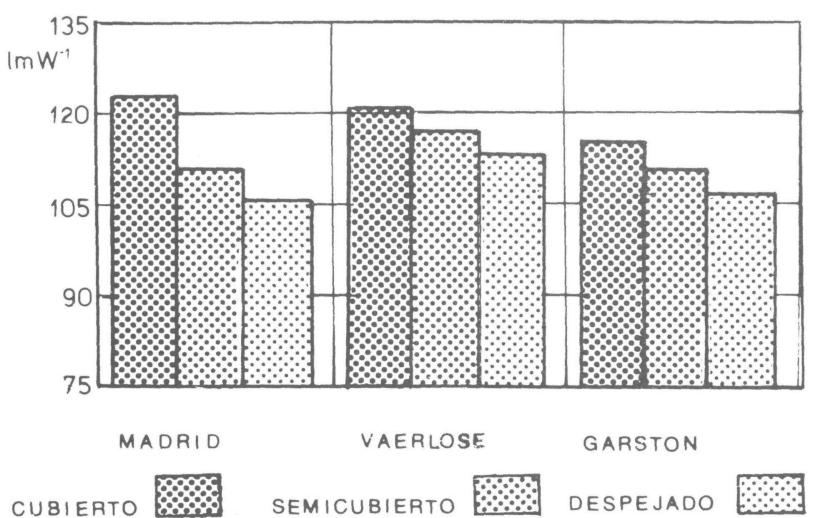

Fig. 3. - Eficacia luminosa de la radiación solar global para distintos lugares y distintas condiciones de cielo.

bosidad de 7 a 8 octavos; y "Semicubierto", cualquier cielo que no cumplía con ninguna de las condiciones anteriores.

Los instrumentos utilizados se muestran en la figura 1. Para medir la iluminancia se utilizó un luxómetro analógico "Evans Electroselenium L.T.D." con lectura para ser transformada en lux (lúmenes $/ \mathrm{m}^{2}$ ) mediante una tabla de conversión; para las medidas de irradiancia se utilizó un piranómetro "Spectrosun" Modelo SR-75, de Spectrolab conectado a un voltímetro, cuyos resultados en voltios fueron transformados en $\mathrm{W} / \mathrm{m}^{2}$ utilizando el factor del piranómetro.

\subsection{Valores medios de la eficacia luminosa}

Los valores medios de la eficacia luminosa para cada uno de los meses, en condiciones de "cielo promedio" (considerando todas las medidas efectuadas con cualquiera de las tres condiciones de cielo) se muestran en la fig. 2. Los valores obtenidos para abril, mayo y junio son respectivamente de 106, 109 y 112 Im W-1. (c) Consejo Superior de Investigaciones Científicas Licencia Creative Commons 3.0 España (by-nc)
En la misma figura están representados los valores obtenidos en otras dos ciudades: Garston $\left(51,71^{\circ} \mathrm{N}\right.$, $0,37^{\circ} \mathrm{W}, 150 \mathrm{~m}$ de altitud) en Gran Bretaña ${ }^{6}$ y Ankara $\left(39,95^{\circ} \mathrm{N}, 32,88^{\circ} \mathrm{E}, 894 \mathrm{~m}\right.$ de altitud) en Turquía ${ }^{7}$. Las mediciones se efectuaron en 1984, en Garston, y en 1980-82, en Ankara; ésta con latitud, condiciones geográficas y clima similares a los de Madrid. Aunque en la figura 2 se han comparado los valores para las tres ciudades, los valores para Garston y Ankara corresponden a los obtenidos de 12 a $13 \mathrm{~h}$ para medidas continuas, mientras que los valores de Madrid se obtuvieron como promedio de varias medidas instantáneas realizadas de las 12 a las $13 \mathrm{~h}$. Se observa que los valores mensuales promedio, que se registraron en Madrid, son del mismo orden que en las otras dos ciudades. Durante los meses citados, y para la hora indicada, la altura solar aumenta al pasar los días. Se observa que para Madrid, de forma similar a lo que ocurre para Garston y Ankara, la eficacia luminosa tiende a aumentar con la altura solar. Se obtuvieron resultados similares utilizando las medidas realizadas a las 9 horas.

En la fig. 3 se indican los valores de eficacia luminosa de la radiación solar global utilizando los datos obtenidos durante el período completo de medidas, considerando por separado las tres condiciones de cielo. Para cielo cubierto, semicubierto y despejado se obtuvieron 123, 111 y $106 \mathrm{~lm} \mathrm{~W} \mathrm{~W}^{-1}$, respectivamente. Se indican también los valores medidos en Vaerlose en Dinamarca ${ }^{1}$ y Garston en Inglaterra, para todo el año.

Se observa que al disminuir la nubosidad los valores decrecen de forma similar en los tres casos. Los valores de la eficacia luminosa de la radiación global con cielo cubierto, semicubierto y despejado, están dentro del rango de valores señalados por Littlefair para diversas localidades ${ }^{1,6}$.

\subsection{Relación de la eficacia luminosa con la altura solar}

Se analizó la variación de la eficacia luminosa en función de la altura solar. Ésta se calculó utilizando la siguiente expresión ${ }^{8}$ :

$$
\gamma=\operatorname{sen}^{-1}(\operatorname{sen} \phi \operatorname{sen} \delta+\cos \omega \cos \phi \cos \delta)
$$

siendo:

$\gamma:$ altura solar en grados,

$\delta$ : declinación en grados,

$\phi$ : latitud en grados,

$\omega$ : ángulo horario, $\omega=(12-t) 15^{\circ}, y$

t: tiempo solar verdadero

http://informesdelaconstruccion.revistas.csic.es 


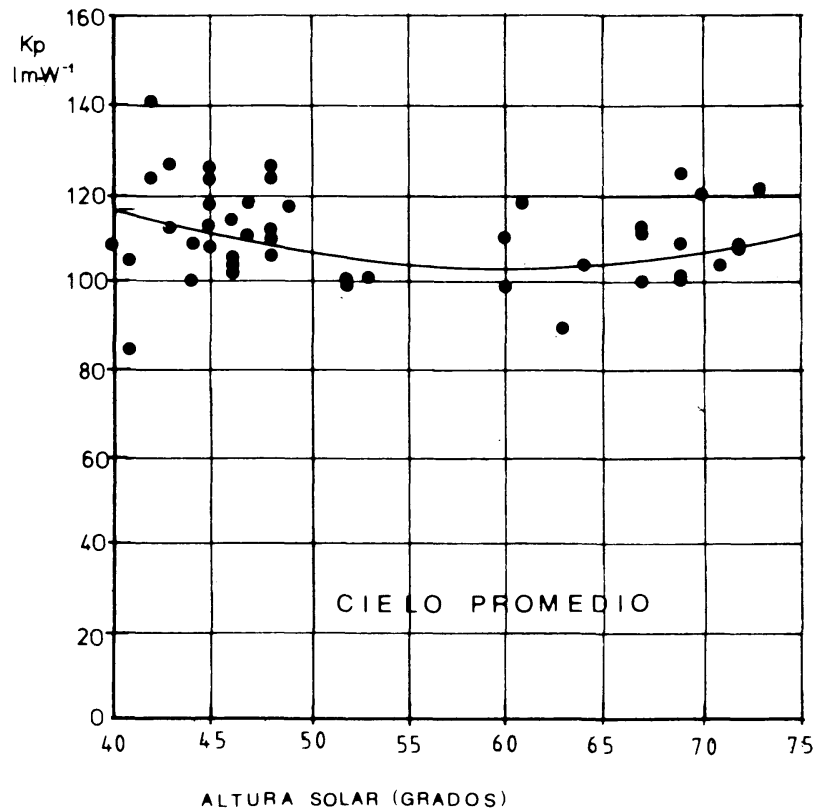

Fig. 4. - Medidas de eficacia luminosa $\left(K_{p}\right)$ de la radiación solar global en condiciones de cielo promedio en función de la altura solar, en Madrid.

\subsubsection{Relación entre la eficacia luminosa y la altura solar para "cielo promedio"}

En la fig. 4 se muestra la relación entre la eficacia luminosa y la altura solar considerando las medidas obtenidas para "cielo promedio".

El mejor ajuste cuadrático, también representado en la figura 4, viene dado por:

$$
\mathrm{K}_{\mathrm{p}}=0,034 \gamma^{2}-4,1 \gamma+228,32\left(\mathrm{Im} \cdot \mathrm{W}^{-1}\right)
$$

donde $\mathrm{K}_{\mathrm{p}}$ es la eficacia luminosa de la radiación solar global con cielo promedio, y $\gamma$ es la altura solar. Para la ecuación [3] se obtuvo un coeficiente de correlación de 0,36 y una desviación estándar de 8,4.

Se consideró conveniente analizar las medidas separadamente, según el tipo de cielo. Se presentan a continuación los resultados obtenidos para cielo despejado y semicubierto. No se presenta un análisis de los datos para cielo cubierto, dado su escaso número.

\subsubsection{Relación entre la eficacia luminosa y la altura solar para cielo despejado}

En este caso la ecuación cuadrática que mejor define la relación entre la eficacia luminosa y la altura solar es la siguiente:

$$
K_{d}=0,005 \gamma^{2}-0,359 \gamma+108,11\left(\mathrm{Im} \cdot \mathrm{W}^{-1}\right)
$$

donde $\mathrm{K}_{\mathrm{d}}$ es la eficacia luminosa de la radiación solar global con cielo despejado, y $\gamma$ es la altura solar. Con

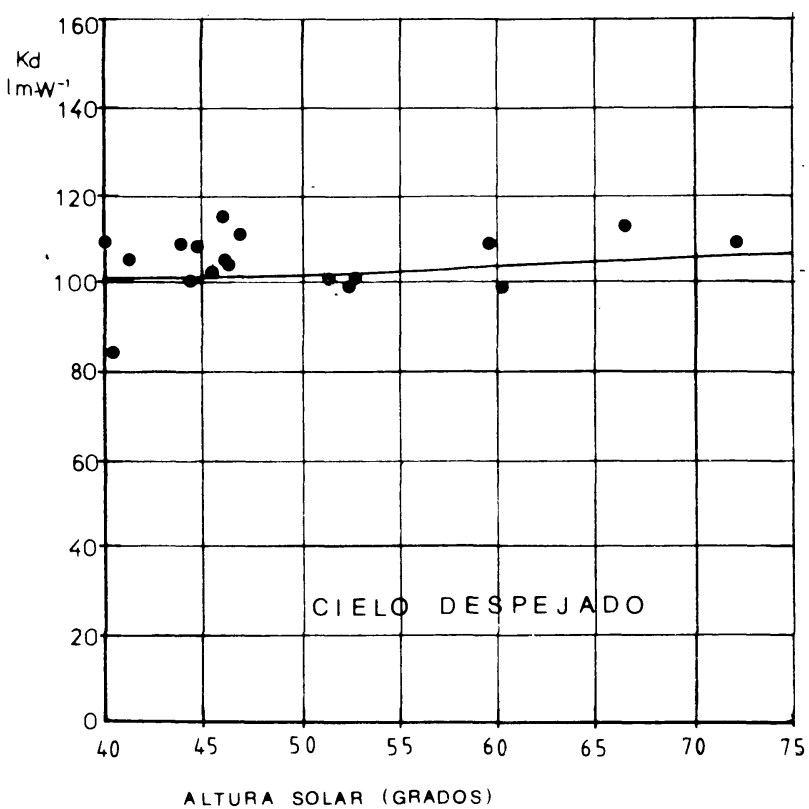

Fig. 5. - Medidas de eficacia luminosa de la radiación solar global en condiciones de cielo despejado $\left(K_{d}\right)$ en función de la altura solar, en Madrid.

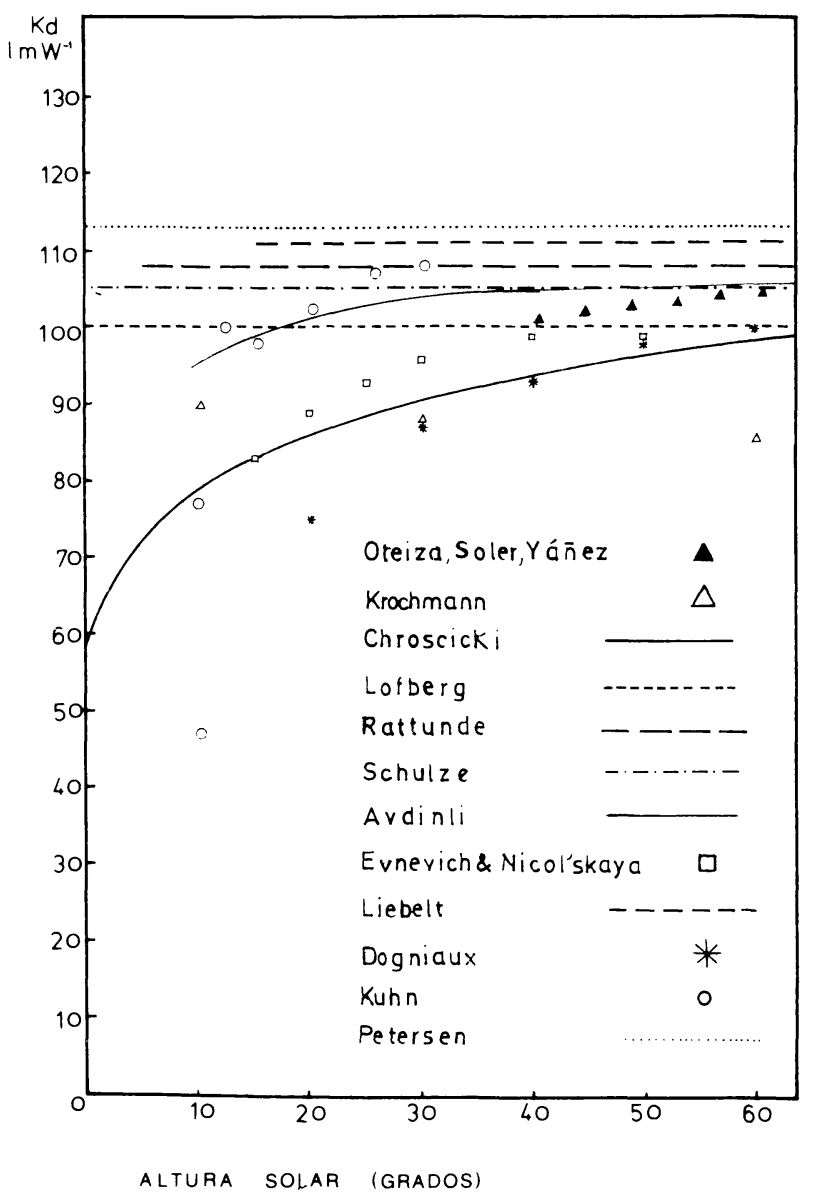

Fig. 6. - Eficacia luminosa de la radiación solar global para cielo despejado $\left(K_{d}\right)$ según varios autores y resultados del presente trabajo. en función de la altura solar. 


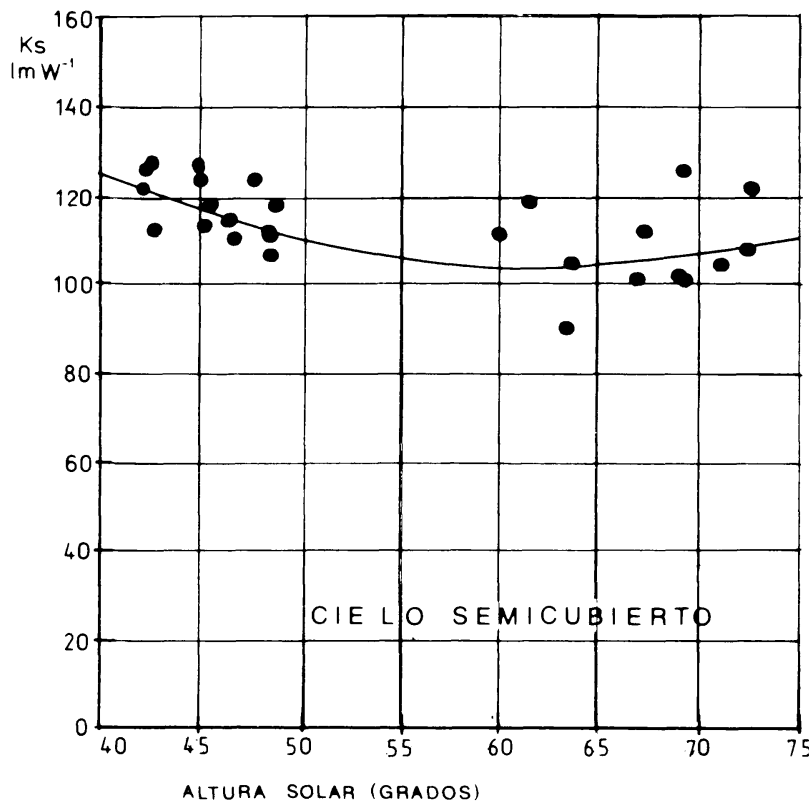

Fig. 7.-Medidas de eficacia luminosa de la radiación solar global en condiciones de cielo semicubierto $\left(K_{s}\right)$ en función de la altura solar, en Madrid.

un coeficiente de correlación de 0,32 y una desviación estándar de 6,9.

En la fig. 5 se muestran las medidas de eficacia luminosa, en función de la altura solar y la relación [4], para estas condiciones de cielo. En la fig. 6 se comparan los resultados de Madrid con los publicados por diversos autores y reseñados por Littlefair ${ }^{6}$.

\subsubsection{Relación entre la eficacia luminosa y la altura solar para cielo semicubierto}

En este caso la ecuación que define con mayor precisión la relación entre la eficacia luminosa con cielo semicubierto $\mathrm{K}_{\mathrm{s}}$ y la altura solar $\gamma$ es la siguiente:

$$
\mathrm{K}_{\mathrm{s}}=0,05 \gamma^{2}-6,14 \gamma+292,57\left(\mathrm{Im} \cdot \mathrm{W}^{-1}\right)
$$

con un coeficiente de correlación de 0,61 y una desviación estándar de 7,7.

En la fig. 7 se muestran las medidas de eficacia luminosa con la altura solar y la relación [5] para estas condiciones de cielo. No comparamos los datos con otros lugares.

Tanto en este caso como en los anteriores se observa una importante dispersión de los resultados experimentales en torno a la curva que constituye el mejor ajuste de éstos. Los bajos coeficientes de correlación obtenidos son habituales en estos experimentos, ya que aparte de los errores experimentales es conocida la dependencia de la eficacia luminosa con diversos parámetros atmosféricos.

\section{CONCLUSIONES}

Los valores medios de la eficacia luminosa para la radiación solar global obtenidos en Madrid, para los meses de abril, mayo y junio de 1989, de 106, 109 y $112 \mathrm{Im} . \mathrm{W}^{-1}$ respectivamente, son similares a los obtenidos para otras localidades europeas.

Los valores de la eficacia decrecen cuando disminuye la nubosidad de forma similar a lo observado en otros lugares, obteniéndose para cielo cubierto, semicubierto y despejado 123, 111 y $106 \mathrm{Im} . \mathrm{W}^{-1}$ respectivamente.

Para cielo despejado la eficacia aumenta ligeramente con la altura solar para $40^{\circ} \leq \gamma \leq 75^{\circ}$, situándose los valores entre los obtenidos por Schulze y Löfberg.

Para cielo semicubierto la eficacia disminuye al aumentar la altura solar, $\gamma$, para $40^{\circ} \leq \gamma \leq 55^{\circ}$.

\section{AGRADECIMIENTO}

Agradecemos al Dr. J. Juan y al Sr. J. M. García, del Instituto de Óptica "Daza de Valdés", del Consejo Superior de Investigaciones Científicas, el apoyo prestado a los autores para la realización del presente trabajo.

\section{BIBLIOGRAFIA}

1. LITTLEFAIR, P.: The luminous efficacy of daylight: a review. Lighting Research and Technology. Vol. 17, N. 4, 162-182, 1985.

2. JUAN, J., PLAZA, L., CRUZ, A.: Variaciones de la iluminancia en Madrid con el día, la hora, y la orientación. Óptica Pura y Aplicada. Vol. 3, 1970.

3. HUNT, DRG.: Availability of daylight. Garston: Building Research Establishment. 1979.

4. OTEIZA, P.: Estudio combinado de iluminación natural y ganancia solar térmica pasiva en edificios docentes. Madrid: Universidad Politécnica, Tesis Doctoral, 1991.

5. CRUZ, A.: Magnitudes fotométricas y radiométricas. Óptica Pura y Aplicada. Vol. 13. 1980.

6. LITTLEFAIR, P.: Measurements of the luminous efficacy of daylight. Lighting Research and Technology. Vol. 20, N. $4,177-188,1988$.

7. HASDEMIR, B.: Daylight availability and energy conservation. Third National Congress on Building Energy Management. Lausana, 361-365, 1987.

8. IGBAL, M.: An introduction to solar radiation. New York: Academic Press, 1983. 